\title{
Precipitação pluviométrica mensal provável em Boa Vista, Estado de Roraima, Brasil
}

\author{
Wellington F. Araújo ${ }^{1}$, Aderson S. de Andrade Júnior², Roberto D. de Medeiros ${ }^{3}$ \& Regynaldo A. Sampaio ${ }^{4}$ \\ 1 UFRR - Departamento de Solos e Irrigação/CCA. BR 174 S/N, Campus do Paricarana, CEP 69310-270, Boa Vista, RR. \\ E-mail: wfaraujo@carpa.ciagri.usp.br (Foto) \\ 2 Embrapa Meio Norte. Av. Duque de Caxias 5650, CEP 64006-220, Teresina, PI. E-mail: aderson@cpamn.embrapa.br \\ ${ }^{3}$ Embrapa/CPAF. BR 174 Km 8, Distrito Industrial, CEP 69301-970, Boa Vista, RR. E-mail: roberto@cpafrr.embrapa.br \\ 4 UFRR. Departamento de Fitotecnia. E-mail: rsampaio@osite.com.br
}

Protocolo $074-28 / 5 / 2001$

\begin{abstract}
Resumo: O objetivo deste trabalho foi de estimar a precipitação mensal provável para o município de Boa Vista, RR ( $2^{\circ} 49^{\prime} 17^{\prime \prime} \mathrm{N} ; 60^{\circ} 39^{\prime} 45^{\prime \prime} \mathrm{W}$ e $90 \mathrm{~m}$ ). Foram utilizados dados de precipitação pluviométrica mensal de 48 anos da série histórica compreendida entre os anos de 1923 a 1997. A estimativa da precipitação mensal provável, em níveis de 10, 20, 25, 30, 50, 60, 70, 75,80 e $90 \%$ de probabilidade, foi obtida utilizando-se as funções de distribuição normal e gama mista. Verificou-se um bom ajuste dos valores mensais de precipitação pluviométrica principalmente à distribuição gama mista, exceto para os meses secos de janeiro e fevereiro. Ficou caracterizada estação chuvosa, compreendida entre os meses de abril e setembro, e seca, entre os meses de outubro e março.
\end{abstract}

Palavras-chave: probabilidade, chuva, planejamento agrícola

\section{Probable monthly rainfall in Boa Vista, Roraima State, Brazil}

\begin{abstract}
This work aimed at estimating the probable monthly rainfall in Boa Vista, RR, $\left(2^{\circ}\right.$ $49^{\prime} 17^{\prime \prime} \mathrm{N}, 60^{\circ} 39^{\prime} 45^{\prime \prime} \mathrm{W}$ e $\left.90 \mathrm{~m}\right)$. The historical data for 48 years from 1923 to 1997 were utilized. Rainfall was estimated using the normal and gamma distribution at 10, 20, 25, 30, 50, $60,70,75,80$ and $90 \%$ probability levels. The gamma distribution provided a good fit, except during the dry months of January and February. The results indicated a rainy season (April September) and a dry season (October - March).
\end{abstract}

Key words: probability, rainfall, agricultural planning

\section{INTRODUÇÃO}

O conhecimento das precipitações prováveis é de extremo interesse para o dimensionamento de vertedouros de barragens, de canais, galerias pluviais, bueiros e barragens de abastecimento de água (Villela \& Mattos, 1975) e para o planejamento agrícola e dimensionamento de sistema de irrigação complementar (Bernardo, 1995). A precipitação provável é a precipitação pluviométrica que apresenta probabilidade específica de ocorrência, baseada em uma longa série de dados (Frizzone, 1979).

No Brasil, a quase totalidade dos projetos de irrigação visa suprir todas as necessidades hídricas da cultura, sem observar a contribuição da precipitação provável do período. Para o adequado planejamento da agricultura, no que se refere ao melhor aproveitamento do clima, é necessário o conhecimento das condições médias, a quantificação da variabilidade e da freqüência de ocorrência de determinados níveis práticos das variáveis climáticas de interesse agrícola com base em uma série histórica de longo período (Berlato, 1992).

Segundo Castro et al. (1994) utiliza-se, no Brasil, a precipitação média no dimensionamento de projetos agrícolas, o que constitui um risco para o produtor. De acordo com Bernardo (1995) para a minimização dos riscos não se deve trabalhar com probabilidades de ocorrência de chuva inferiores a 75 ou $80 \%$. Entretanto, esses valores podem ser variáveis adotando-se um critério econômico, cujo nível de probabilidade esteja associando à redução na qualidade e quantidade de produção, decorrentes da deficiência hídrica pela falta de chuva, durante determinado período (Jensen, 1983).

Vários modelos de distribuição de freqüência têm sido utilizados, visando a estimativa da precipitação provável para diferentes locais e períodos de ocorrência. Dentre eles, destacam-se as distribuições normal (Frizzone, 1979; Assis et al., 1996; Andrade Júnior \& Bastos, 1997 ) e gama (Frizzone, 1979; Castro Neto \& Silveira, 1983; Assis et al., 1996; Rodrigues \& Pruski, 
1996, Ribeiro \& Lunardi, 1997; Andrade Júnior \& Bastos, 1997). Para o caso específico do município de Boa Vista em Roraima, Medina \& Leite (1984) efetuaram estimativas da probabilidade de precipitações mensais através do método de Kimball. Entretanto, Pinto et al. (1976) salientam que embora o método de Kimball possa ser utilizado para séries totais, os dados necessitam ser ajustados por uma função estatística e se prestam mais para dados extremos. Os autores recomendam o uso de funções probabilísticas fazendo-se, posteriormente, a verificação do ajuste através de testes de aderência.

Além do conhecimento da precipitação pluvial, é importante conhecer o balanço hídrico climático, o qual indica a disponibilidade de água para as plantas em dado tipo de solo. Neste sentido, a metodologia mais utilizada para fins agroclimatológicos é a desenvolvida por Thornthwaite \& Mather (1955) que consiste no balanço entre a precipitação e a evapotranspiração potencial para determinada capacidade de água disponível (CAD).

Com o presente trabalho objetivou-se estimar a precipitação pluviométrica mensal em vários níveis de probabilidade para a região de cerrado em Boa Vista, Estado de Roraima, e determinar o período de irrigação para uma CAD de $25 \mathrm{~mm}$, considerando-se apenas os dados de precipitação com probabilidade de $80 \%$ de ocorrência.

\section{MATERIAL E MÉTODOS}

Foram utilizados os dados mensais de precipitação pluviométrica do município de Boa Vista, Estado de Roraima, obtidos junto à Delegacia Federal de Agricultura do Estado, cujas coordenadas geográficas de referência são: $2^{\circ} 49^{\prime} 17^{\prime \prime} \mathrm{N}$, $60^{\circ} 39^{\prime} 45^{\prime \prime} \mathrm{W}$ e $90 \mathrm{~m}$. A série histórica compreendeu o período entre os anos de 1923 e 1997, sendo excluídos aqueles com dados incompletos, o que resultou em um aproveitamento de 48 anos de dados. A estimativa de precipitação pluviométrica mensal provável foi obtida para os níveis de $10,20,25,30,50$, $60,70,75,80$ e $90 \%$ de probabilidade, utilizando-se as funções de distribuição normal e gama, conforme apresentado por (Assis et al., 1996).

A distribuição gama de probabilidade é a mais utilizada para ajustar totais de chuva de períodos mensais ou menores. Sua função densidade de probabilidade apresenta a seguinte forma:

$$
F(X)=\frac{1}{\Gamma(\alpha) \beta^{\alpha}} \int_{0}^{X} X^{\alpha-1} e^{-\frac{X}{\beta}} d(X)
$$

As estimativas dos parâmetros $\alpha$ e $\beta$ foram efetuadas pelo método da máxima verossimilhança, através das seguintes expressões (Assis et al., 1996):

$$
\begin{gathered}
\alpha=\frac{1}{4 \mathrm{~A}}\left(1+\sqrt{1+\frac{4 \mathrm{~A}}{3}}\right) \\
\beta=\frac{\overline{\mathrm{X}}}{\alpha}
\end{gathered}
$$

sendo:

$$
\begin{gathered}
\mathrm{A}=\ln \overline{\mathrm{X}}-\mathrm{X}_{\mathrm{g}} \\
\overline{\mathrm{X}}=\frac{1}{\mathrm{~N}} \sum_{\mathrm{i}=1}^{\mathrm{N}} \mathrm{X}_{\mathrm{i}} \\
\mathrm{X}_{\mathrm{g}}=\frac{1}{\mathrm{~N}} \sum_{\mathrm{i}=1}^{\mathrm{N}} \ln \left(\mathrm{X}_{\mathrm{i}}\right)
\end{gathered}
$$

em que:

$$
\begin{array}{ll}
\bar{X} & \text { - média aritmética das observações } \\
\mathrm{X}_{\mathrm{g}} & \text { - média geométrica das observações } \\
\mathrm{N}^{-} & \text {- número de observações }
\end{array}
$$

Cunha et al. (1996) sugerem que duas situações distintas podem ocorrer, quando se utiliza a distribuição gama: a) a série de dados não contém valores nulos; neste caso, a estimativa da freqüência de ocorrência é obtida através da distribuição cumulativa gama, sendo possível a estimativa dos parâmetros da distribuição $(\alpha$ e $\beta)$, pelo método da máxima verossimilhança; b) a série contém valores nulos; caso em que se utiliza a distribuição cumulativa gama mista, a qual é determinada em duas partes, da seguinte forma (Assis et al., 1996):

$$
\mathrm{F}(\mathrm{X})=\mathrm{P}_{0}+\left(1-\mathrm{P}_{0}\right) \mathrm{G}(\mathrm{X})
$$

sendo:

$$
\mathrm{P}_{0}=\frac{\mathrm{N}_{0}}{(\mathrm{~N}+1)}
$$

em que:

$\mathrm{P}_{0} \quad$ - probabilidade de ocorrência de valores nulos

$G(X)$ - distribuição cumulativa gama

$\mathrm{N}_{0} \quad$ - número de valores nulos da série

Para a função de probabilidade normal ou curva de Gauss, utilizou-se a seguinte forma normal reduzida abaixo (Assis et al., 1996):

$$
f(X)=\frac{1}{\sqrt{2 \pi}} \int_{-\infty}^{Z} e-\frac{Z^{2}}{2}
$$

$$
\mathrm{Z}=(\mathrm{X}-\overline{\mathrm{X}}) / \mathrm{S}
$$

em que:

$$
\begin{aligned}
& \bar{X} \text { - média } \\
& S \text { - variância }
\end{aligned}
$$

Para estimativa dos valores de precipitação provável, utilizou-se a planilha eletrônica Excel, a qual retorna o inverso da distribuição acumulada normal e gama, a partir dos valores de média $(\mu)$, desvio-padrão $(\sigma), \alpha, \beta$ e dos níveis de probabilidades de $10,20,25,30,40,50,60,70,75,80$ e $90 \%$. 
Tabela 1. Médias mensais, desvio-padrão, coeficiente de variação (CV) e valores extremos de precipitação pluviométrica e ano de ocorrência em Boa Vista, RR, para o período de 1923 a 1997

\begin{tabular}{|c|c|c|c|c|c|c|c|}
\hline \multirow[t]{2}{*}{ Mês } & Média & Desvio Padrão & \multirow{2}{*}{$\begin{array}{l}\mathrm{CV} \\
(\%)\end{array}$} & \multirow{2}{*}{$\begin{array}{l}\text { Mínimo } \\
\text { (mm) }\end{array}$} & \multirow{2}{*}{$\begin{array}{c}\text { Ano } \\
\text { Ocorrido }\end{array}$} & \multirow{2}{*}{$\begin{array}{l}\text { Máximo } \\
\text { (mm) }\end{array}$} & \multirow{2}{*}{$\begin{array}{c}\text { Ano } \\
\text { Ocorrido }\end{array}$} \\
\hline & & $\mathrm{mm}$ & & & & & \\
\hline Janeiro & 29,3 & 38,8 & 132,3 & 0,0 & 1994 & 207,2 & 1950 \\
\hline Fevereiro & 24,7 & 31,7 & 128,1 & 0,0 & 1985 & 130,8 & 1950 \\
\hline Março & 47,8 & 59,5 & 124,6 & 0,0 & 1954 & 299,5 & 1979 \\
\hline Abril & 122,2 & 98,3 & 80,5 & 0,0 & 1925 & 456,8 & 1976 \\
\hline Maio & 291,2 & 109,7 & 37,7 & 18,8 & 1974 & 484,7 & 1942 \\
\hline Junho & 352,9 & 123,6 & 35,0 & 42,2 & 1979 & 638,2 & 1996 \\
\hline Julho & 335,1 & 121,4 & 36,2 & 31,4 & 1983 & 605,4 & 1947 \\
\hline Agosto & 209,7 & 96,4 & 46,0 & 81,2 & 1930 & 458,0 & 1954 \\
\hline Setembro & 97,1 & 59,9 & 61,7 & 0,4 & 1997 & 251,0 & 1974 \\
\hline Outubro & 67,6 & 66,5 & 98,5 & 0,0 & 1997 & 321,2 & 1934 \\
\hline Novembro & 56,8 & 46,0 & 80,9 & 0,0 & 1947 & 161,0 & 1954 \\
\hline Dezembro & 44,2 & 46,1 & 104,3 & 0,0 & 1946 & 181,9 & 1927 \\
\hline Anual & 1678,6 & 397,1 & 23,5 & 994,7 & & 2554,6 & \\
\hline
\end{tabular}

Para se verificar o ajuste dos dados pluviométricos mensais à função de distribuição gama mista e normal, foi utilizado o teste de aderência de Kolmogorov-Smirnov, considerando-se um nível de $5 \%$ de probabilidade.

O balanço hídrico climático, foi calculado de acordo com Thornthwaite \& Mather (1955) e para estimativa da evapotranspiração potencial foi utilizada a equação proposta por Thornthwaite (1948) considerando-se uma CAD de $25 \mathrm{~mm}$.

\section{RESULTADOS E DISCUSSÃO}

De acordo com a série climatológica observou-se, para Boa Vista, RR, precipitação anual média de 1688,4 mm, sendo 1945 o ano mais chuvoso, com 2554,6 mm, e 1983 o ano menos chuvoso, com 994,7 mm. Os coeficientes de variação das médias mensais e o desvio-padrão foram elevados, evidenciando-se a grande variabilidade da precipitação, sendo que as variações menores ocorreram entre os meses de maio a agosto, e as maiores entre os meses de dezembro a março que correspondem ao período mais chuvoso e mais seco, respectivamente (Tabela 1); assim, sob o ponto de vista estatístico, as flutuações nos valores de precipitação média mensal mostraram ser inadequadas para o planejamento agrícola, motivando estudos probabilísticos. Como foram observados valores nulos durante o período chuvoso da série histórica, utilizou-se a distribuição gama mista e a normal, conforme recomendação de Assis et al. (1996).

Com base nos parâmetros $\alpha$ e $\beta$, estimados pelo método da verossimilhança, verificou-se a possibilidade do uso da distribuição gama mista (Tabela 2). Os valores de $\alpha$ não excederam o valor de 100 em nenhum mês, possibilitando o uso da distribuição de probabilidade de chuva selecionada. Segundo Thom (1958) valores de $\alpha$ superiores a 100, indicam que a distribuição gama incompleta não deve ser utilizada.

Tabela 2. Parâmetros $\alpha$ e $\beta$ da distribuição gama calculados pelo método da máxima verossimilhança para a precipitação pluviométrica mensal em Boa Vista, RR

\begin{tabular}{lcc}
\hline Meses & $\alpha$ & $\beta$ \\
\hline Janeiro & 0,8212 & 58,1582 \\
Fevereiro & 1,2269 & 99,5672 \\
Março & 4,3276 & 67,2884 \\
Abril & 6,4227 & 54,9477 \\
Maio & 5,8270 & 57,5079 \\
Junho & 4,8125 & 43,5793 \\
Julho & 1,5379 & 63,1357 \\
Agosto & 1,3924 & 48,5357 \\
Setembro & 1,5957 & 35,6123 \\
Dezembro & 1,2558 & 35,2177 \\
\hline
\end{tabular}

Na Tabela 3 são apresentados os dados de precipitação pluviométrica mensal em diferentes níveis de probabilidades segundo a função de distribuição gama mista e a média aritmética de cada mês. Foi obtido um ajuste adequado dos valores mensais de precipitação pluviométrica à distribuição gama mista para o total anual, e para os meses de março a dezembro, que correspondem aos meses de maiores

Tabela 3. Precipitação pluviométrica $(\mathrm{mm})$ mensal e anual provável para o município de Boa Vista, RR, em diferentes níveis de probabilidade, segundo a função de distribuição gama mista

\begin{tabular}{|c|c|c|c|c|c|c|c|c|c|c|c|c|c|}
\hline$\%$ & Jan & Fev & Mar & Abr & Mai & Jun & Jul & Ago & Set & Out & Nov & Dez & Anual \\
\hline 90 & 5,1 & 2,1 & 3,2 & 17,7 & 132,3 & 190,3 & 174,0 & 100,3 & 19,5 & 11,3 & 11,0 & 6,3 & 1199,1 \\
\hline 80 & 8,6 & 4,3 & 7,7 & 33,5 & 171,8 & 233,7 & 216,3 & 128,1 & 33,2 & 20,0 & 18,5 & 11,7 & 1345,0 \\
\hline 75 & 10,3 & 5,5 & 10,4 & 41,6 & 188,8 & 251,8 & 234,1 & 139,9 & 39,9 & 24,4 & 22,1 & 14,5 & 1403,5 \\
\hline 70 & 12,1 & 6,9 & 13,3 & 50,0 & 205,0 & 268,9 & 250,9 & 151,2 & 46,7 & 28,9 & 25,7 & 17,3 & 1457,4 \\
\hline 60 & 15,8 & 9,9 & 20,2 & 68,3 & 236,7 & 301,7 & 283,4 & 173,1 & 61,1 & 38,4 & 33,4 & 23,5 & 1558,3 \\
\hline 50 & 20,0 & 13,5 & 28,6 & 89,2 & 269,1 & 334,8 & 316,1 & 195,4 & 77,1 & 49,1 & 41,9 & 30,6 & 1656,8 \\
\hline 40 & 24,8 & 18,0 & 39,1 & 114,1 & 304,4 & 370,2 & 351,4 & 219,6 & 95,6 & 61,8 & 51,7 & 39,0 & 1759,3 \\
\hline 30 & 30,8 & 23,8 & 53,1 & 145,6 & 345,4 & 410,7 & 391,8 & 247,5 & 118,6 & 77,5 & 63,9 & 49,5 & 1873,6 \\
\hline 25 & 34,6 & 27,5 & 62,1 & 165,3 & 369,6 & 434,3 & 415,5 & 264,0 & 132,8 & 87,2 & 71,3 & 56,1 & 1939,0 \\
\hline 20 & 39,0 & 32,0 & 73,3 & 189,1 & 397,9 & 461,7 & 442,9 & 283,1 & 149,8 & 99,0 & 80,3 & 64,1 & 2013,5 \\
\hline 10 & 52,4 & 46,2 & 108,5 & 262,0 & 478,8 & 539,0 & 520,7 & 337,7 & 201,1 & 134,7 & 107,2 & 88,5 & 2218,5 \\
\hline Média & 29,3 & 24,7 & 47,8 & 122,2 & 291,2 & 352,9 & 335,1 & 209,7 & 97,1 & 67,6 & 56,8 & 44,2 & 1678,6 \\
\hline
\end{tabular}



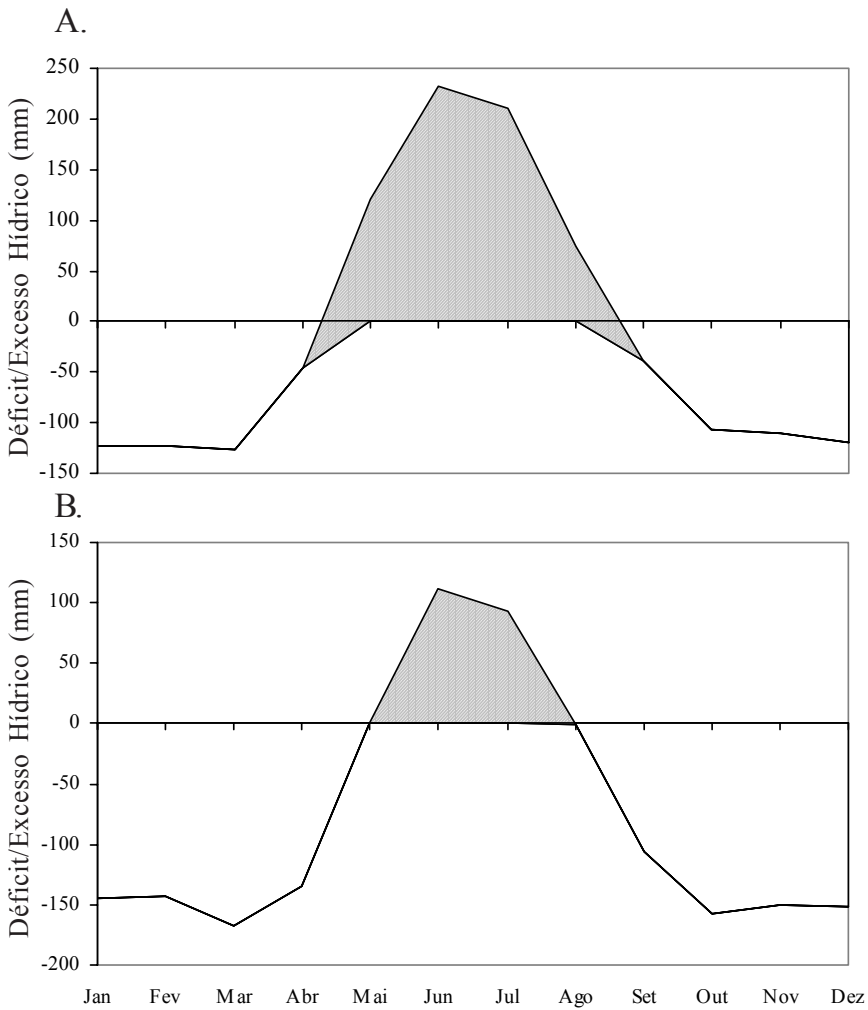

Figura 1. Extrato do balanço hídrico climático mensal para Boa Vista, RR, calculado pelo método de Thornthwaite \& Mather (1955), utilizando-se capacidade de água disponível $25 \mathrm{~mm}$, considerando-se a precipitação normal (A) e a probabilidade de $80 \%$ (B)

precipitações. Estes resultados concordam com aqueles obtidos por Medina \& Leite (1984) para mesma região.

Exceto para janeiro e fevereiro, período mais seco, as precipitações nos demais meses ajustaram-se à função de distribuição gama mista. Os resultados estão de acordo com Rodrigues \& Pruski (1996) e Andrade Júnior \& Bastos (1997) onde os períodos secos também não se ajustaram a distribuição gama. No geral, o valor médio mensal de precipitação pluviométrica ocorreu entre os níveis de 30 e $40 \%$ de probabilidade, exceto para os meses de maior precipitação (maio a agosto) cujos níveis se situam entre 40 e 50\% (Tabela 3). Resultados semelhantes foram obtidos por Ribeiro \& Lunardi (1997) para a região de Lavras, MG, e Andrade Júnior \& Bastos (1997) para a região do cerrado do Piauí, enquanto Castro Neto \& Silveira (1983) encontraram também em Lavras, para precipitação média valores de probabilidade variando de 21 a $42 \%$. Desta forma, o uso do valor médio de precipitação como base em projetos agrícolas deve ser evitado.

Pela função normal, houve ajuste dos meses de abril a novembro e para o total anual; entretanto, o ajuste das precipitações mensais à curva de Gauss foi inferior ao ajuste dos dados à curva de distribuição gama. Resultados idênticos foram observados por Andrade Júnior \& Bastos (1997) razão por que se recomenda o uso da distribuição gama em estudos visando à estimativa da precipitação provável.

Observou-se que o período chuvoso se estendeu de meados de abril a meados de setembro, considerando-se os dados normais de precipitação (Figura 1A). Embora essencial, a definição da estação chuvosa não é, por si só, a única a ser observada para o plantio de sequeiro. Outros aspectos devem ser observados, tais como: características hídricas do solo, cultura a ser explorada, práticas culturais adotadas e presença de períodos de estiagem (veranicos) dentro do período chuvoso.

Segundo Bernardo (1995) o nível de 75 a 80\% de probabilidade de ocorrência de chuva é o mais confiável para dimensionamento de projetos agrícolas ou de irrigação. Assim, calculou-se o balanço hídrico considerando-se uma probabilidade de precipitação de $80 \%$ e uma CAD de $25 \mathrm{~mm}$ (Figura 1B), obtendo-se o período viável para agricultura de sequeiro de ciclo curto, que se estende por 4 meses. Nos outros 8 meses do ano, para que a exploração agrícola seja viável na região, haveria a necessidade do uso da irrigação integral e/ou complementar. É importante ressaltar que os solos de cerrado de Roraima apresentam baixa capacidade de retenção de água (Lopes, 1983) e fertilidade natural (EMBRAPA, 1992) sendo necessário uma análise econômica, antes da adoção de um pacote tecnológico que inclua o uso da irrigação.

\section{CONCLUSÕES}

Os resultados obtidos neste trabalho permitiram concluir-se que:

1. O período de maior precipitação pluviométrica em Boa Vista, RR, tem início em abril e se prolonga até setembro, indicando um período viável de cultivo por seis meses com uso eventual de irrigação complementar.

2. Nos meses de junho e julho ocorrem as maiores precipitações do ano com médias superiores a $330 \mathrm{~mm}$; e, considerando-se $80 \%$ de probabilidade de precipitação, há somente quatro meses (maio, junho, julho e agosto) cuja precipitação atende à demanda hídrica das culturas, sem necessidade de irrigação.

3. Os resultados de precipitação ajustam-se melhor a distribuição de probabilidade gama.

\section{LITERATURA CITADA}

Andrade Júnior, A.S. de; Bastos, E.A. Precipitação pluviométrica provável em municípios do cerrado piauiense. Teresina: EMPRABA-CPAMN, 1997. 22p. Documentos, 25

Assis, F.N.; Arruda, H.V. de; Perreira, A.R. Aplicações de estatística à climatologia: teoria e prática. Pelotas: Ed. Universitária UFPel, 1996. 161p.

Berlato, M.A. As condições de precipitação pluvial no Estado do Rio Grande do Sul e os impactos das estiagens na produção agrícola. In: Bergamaschi, H. Agrometeorologia aplicada à irrigação. Porto Alegre: Ed. Universitária UFRGS, 1992. cap.1, p.11-24.

Bernardo, S. Manual de irrigação. 6.ed. Viçosa: Imprensa Universitária, 1995.657p.

Castro, L.H.R.; Moreira, A.N.; Assad, E.D. Definição e regionalização dos padrões pluviométricos dos cerrados brasileiros. In: Assad, E.D. (coord.) Chuvas no cerrados: análise e espacialização. Brasília: Embrapa, CPAC; Embrapa, SPI, 1994. p.13-23. 
Castro Neto, P.; Silveira, J.V. Precipitação provável para LavrasMG, baseada na função de distribuição de probabilidade gama. III Períodos de 10 dias. Ciência e Prática, Lavras, v. 7 , p.58-65. 1983.

Cunha, A.R.; Martins, D.; Passos, J.R.S. O modelo Gama de probabilidade aplicado ao estudo da distribuição da chuva na região administrativa de Bauru, SP. In: Congresso Brasileiro de Engenharia Agrícola, 25, Bauru, 1996. Anais... Bauru: Sociedade Brasileira de Engenharia Agrícola, 1996. CDRom

EMBRAPA - Empresa Brasileira de Pesquisa Agropecuária Centro de Pesquisa Agroflorestal de Roraima. Síntese da pesquisa agropecuária em Roraima. Boa Vista, RR. 1992. $60 p$.

Frizzone, J.A. Análise de cinco modelos para o cálculo da distribuição e freqüência de precipitação na região de Viçosa, MG. Viçosa: UFV, 1979, 100p. Dissertação Mestrado

Jensen, M.E. Design and operation of farm irrigation system. New York: ASAE. 1983. 829p.

Lopes, A.S. Solos sob "cerrado", características, propriedades e manejo. Piracicaba: Instituto Potassa \& Fosfato, 1983. 162p.
Medina, B.F.; Leite, J.A. Probabilidade de chuvas em Boa Vista, RR. Pesquisa Agropecuária Brasileira, Brasília, v.19, p.1437-1441. 1984.

Pinto, N.L. de; Holtz, A.C.T.; Martins, J.A.; Gomide, F.L.S. Hidrologia básica. São Paulo: Ed. Edgard Blücher. 1976. 278p

Ribeiro, A.M. de A.; Lunardi, C. A precipitação mensal provável para Londrina, PR, através da função gama. Energia na Agricultura, Botucatu, v.12, p.37-44. 1997.

Rodrigues, L.N.; Pruski, F.F. Precipitação provável para João Pinheiro, Minas Gerais, utilizando funções de distribuição de probabilidade gama e log-normal. In: Congresso Brasileiro de Engenharia Agrícola, 25, Bauru,1996. Anais... Bauru: Sociedade Brasileira de Engenharia Agrícola, 1996. CD-Rom

Thom, H.C.S. A note on the gamma distribution. Monthly weather Review. Washington, v.86, p.117-122. 1958.

Thornthwaite, C.W. An approach toward a rational classification of climate. Geographic Review, New Jersey, v.38, p.55-94, 1948.

Thornthwaite, C.W; Mather, J.R. The water balance. New Jersey: Drexel Institute of Technology, 1955. 104p.

Villela, S.M.; Mattos, A. Hidrologia aplicada. São Paulo: McGraw-Hill do Brasil, 1975. 245p. 